accumulate within the larynx and trachea, above the cuff. When the cuff is deflated, these secretions may contaminate the proximal trachea and bronchial tree. The LMA protects against this. Finally, the LMA avoids tracheal intubation, which may be technically demanding in this patient group.

Case selection is important. Use of the LMA is inappropriate when a patient has a full stomach, low respiratory compliance (leading to high inflation pressures during mechanical ventilation) or periglottic pathology which interferes with correct placement of the LMA.

LMA for tracheostomy was originally described in 1992, ${ }^{1}$ and a case series reported recently. ${ }^{2}$ We find that the flexible ('re-inforced') LMA is the most suitable because the flexible nature of the airway tube allows it (and the attached breathing system) to be positioned out of the operative field.

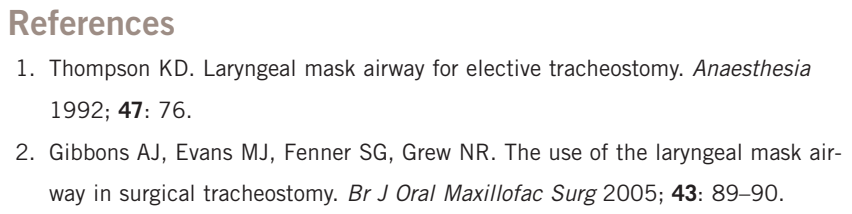

\section{COMMENT 2}

\section{doi $10.1308 / 003588406 \times 106397$}

\section{Percutaneous tracheostomy}

\section{MARTIN H THOMAS}

Department of Vascular Surgery, St Peter's Hospital, Chertsey, Surrey, UK

\section{CORRESPONDENCE TO}

Mr MH Thomas, Department of Vascular Surgery, St Peter's Hospital, Guilford Road, Chertsey, Surrey KT16 OPZ, UK.

E: martin.thomas@doctors.org.uk

I enjoyed this article on surgical tracheostomy describing the authors' obviously excellent, meticulous and elegant technique. However, there is no doubt in my mind that the procedure of choice in the intensive care unit (ITU), where most tracheostomies are performed, is the percutaneous method.

The authors suggest that the surgical procedure should be carried out in an operating theatre, and herein lies the problem. ITU patients are like Algerian wine - they do not travel well. Moving such a patient to the operating theatre from ITU can be a major undertaking, requiring an anaesthetist, an ODP, a nurse, a porter and perhaps even a distraught relative. For this reason, I carried out 104 consecutive surgical tracheostomies at the bedside in ITU, rather than in theatre. The wide bed should not put the surgeon off. The procedure still requires instruments brought from theatre, a diathermy machine, a dedicated sucker, proper lighting, a nurse, an ODP and perhaps an assistant. There was one death directly attributable to the operation; minor bleeding and postoperative infection were common.

I then switched to percutaneous tracheostomy, having been trained by an ITU consultant colleague in another hospital. The technique is quicker and easier, requires no extra instruments, no assistant and no operative surgical skills. In a personal series of 94, one patient died from a cardiac arrest as the procedure started. Minor complications and infective problems have been minimal. Furthermore, most of the percutaneous tracheostomies are now performed by the ITU staff. Since introducing percutaneous tracheostomy, we have only required to perform one open surgical tracheostomy on an ITU or HDU patient and this was done for us by an ENT surgeon.

\section{AUTHOR'S RESPONSE}

\section{doi 10.1308/003588406X106405 JPM PRACY}

Department of Otolaryngology, The Queen Elizabeth Hospital, Edgbaston, Birmingham, UK

\section{CORRESPONDENCE TO}

Mr JPM Pracy, Consultant Otolaryngologist/Head \& Neck Surgeon, The Queen Elizabeth Hospital, Edgbaston, Birmingham B15 2TH, UK. E: lucie.wright@uhb.nhs.uk

We are in agreement with Mr Thomas as to the performance of percutaneous tracheostomy at the bedside for simple, straight-forward ITU cases and indeed there is extensive literature to support this point of view.

It is certainly the case that it is preferable not to transfer patients from ITU to the operating theatre. However, the majority of patients who now undergo a formal, open, surgical tracheotomy are patients with complex anatomy or medical problems and we feel that these are best dealt with in the environment of an operating theatre.

Having said this, as is always the case, a risk-benefit analysis needs to be carried out in one's own mind as to the risks of patient transfer against the risks of complications during a procedure that is being carried out in a less than ideal environment. It is certainly possible to carry out a tracheotomy at the bedside in ITU; however, the width of the bed is not to be underestimated as this requires the surgeon to lean over much further than is normally the case, resulting in low back pain, and it may indeed be the case that there are health and safety issues for the surgeon and/or assistant.

We do not feel that people should be proscriptive about either the technique or place where the tracheostomy is carried out. 